\title{
Ultracold atoms in optical lattices with random on-site interactions
}

\author{
H. Gimperlein ${ }^{1}$, S. Wessel ${ }^{1}$, J. Schmiedmayer ${ }^{2}$, and L. Santos ${ }^{1}$ \\ (1) Institut für Theoretische Physik III, Universität Stuttgart, Pfaffenwaldring 57 V, D-70550 Stuttgart \\ (2) Physikalisches Institut, Universität Heidelberg, Philosophenweg 12, D-69120 Heidelberg
}

\begin{abstract}
We consider the physics of lattice bosons affected by disordered on-site interparticle interactions. Characteristic qualitative changes in the zero temperature phase diagram are observed when compared to the case of randomness in the chemical potential. The Mott-insulating regions shrink and eventually vanish for any finite disorder strength beyond a sufficiently large filling factor. Furthermore, at low values of the chemical potential both the superfluid and Mott insulator are stable towards formation of a Bose glass leading to a possibly non-trivial tricritical point. We discuss feasible experimental realizations of our scenario in the context of ultracold atoms on optical lattices.
\end{abstract}

PACS numbers: 03.75.Ss,05.30.Fk

Ultracold atomic gases have attracted large interest during the last years, in particular due to the experimental achievement of quantum degeneracy both for bosonic 1] and fermionic 2] gases. Very recently, the extraordinary experimental developments in the manipulation of ultracold atoms have opened the path towards a new fascinating research area, namely the analysis of stronglycorrelated atomic gases. In this sense, remarkable experimental results have been already reported, including experiments on the so-called BEC-BCS crossover in degenerate Fermi gases [3], and experiments on ultracold gases in optical lattices, such as e.g. the observation of the Mott insulator-to-superfluid transition [4, 5] and the experimental realization of a Tonks-Girardeau gas $[6]$.

Up to now the experiments performed in optical lattices have considered a defect-free square lattice potential. However, recent proposals have discussed new scenarios which depart from this standard. In particular, various lattice geometries are achievable by standard laser techniques, and should lead to fascinating physics [7, 8]. On the other hand, disorder may be induced in the laser potential in different ways. Localized impurities can be created, leading to Kondo-like physics [9]. Randomness can be produced by means of additional incommensurable lattices, or by laser speckles [10], and may lead to Anderson localization [1] and Bose glass phases [12]. First experimental steps towards random potentials have already been achieved 13. Additionally, randomness may be induced in the hopping rates in the optical lattice, leading to Fermi glasses, spin glasses and quantum percolation [14].

One of the most fascinating possibilities for the control of atomic gases is provided by the manipulation of the interatomic interactions by means of Feshbach resonances [15]. Using a suitably adjusted magnetic field a resonance is induced between a molecular state and the unbound states of the incoming atoms, leading to important modifications of the scattering properties. The $s$-wave scattering length undergoes a very large change in a relatively narrow window of values of the applied magnetic field, becoming $\pm \infty$ at the resonance. This ex- treme sensitivity of the scattering properties at the verge of a Feshbach resonance is crucial for our discussion.

In this Letter, we consider the physics of lattice bosons subject to a novel kind of disorder, namely bounded disorder in the strength of the interatomic interactions [16]. In particular, we show how this type of disordered system can be realized in the context of ultracold atoms near a Feshbach resonance. We discuss the consequences that the disorder in the interaction strength has on the zerotemperature phase diagram of the system by means of a strong-coupling expansion (SCE) and quantum Monte Carlo (QMC) simulations, and contrast our findings to the case of randomness in the chemical potential [12].

The above scenario can be realized in a gas of ultracold bosons confined to an optical lattice using state-of-theart experimental techniques on atom chips [17]. In the following, we consider a one-dimensional configuration, where the atoms are assumed to be strongly confined in the other two dimensions (by e.g. additional optical confinement). However, qualitatively similar results as those discussed in this Letter also hold in higher dimensions. We assume that the gas is brought at the verge of a Feshbach resonance by an off-set magnetic field, where, as discussed previously, slight modifications of the magnetic field lead to large variations of the scattering properties. We furthermore consider the bosonic gas to be close to a magnetic wire, inducing a spatially random magnetic field [17], which can be considered sufficiently weak, such that the variations in the potential energy can be considered negligible when compared to other energy scales. However, since the off-set field sets the system at the verge of a Feshbach resonance, the slight random variations of the magnetic field lead to a spatially random variation of the local interatomic interactions (see Fig. (1). We assume that the variations of the scattering properties are bounded, i.e. the spatial variations of the magnetic field do not induce a crossing of the resonance.

In order to avoid significant van der Waals losses, the atoms should be placed at a distance $>0.5 \mu \mathrm{m}$ from the surface of the atom chip, which restricts the characteristic wavelength of the variations of the magnetic field 


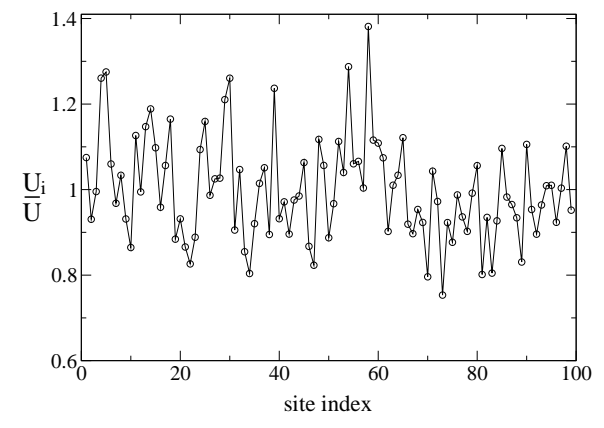

FIG. 1: Typical spatial variation of the on-site interactions. In the figure we have considered a ${ }^{87} \mathrm{Rb}$ cloud $0.5 \mu \mathrm{m}$ over the surface of an atom chip, at a $1 \mu \mathrm{m}$-wide magnetic wire with a current leading to a magnetic field variation of up to $\pm 2.5 \mathrm{mG}$, an off-set field $5 \mathrm{mG}$ away from the $685.8 \mathrm{G}$ resonance, a lattice spacing of $1 \mu$, and a lattice depth of 5 recoil energies. $U_{i}$ is obtained by integrating $\int d x U(x)\left|\psi_{i}(x)\right|^{4} / \int d x\left|\psi_{i}(x)\right|^{4}$, with $\psi_{i}(x)$ the $i$-th on-site wavefunction which is approximated by a Gaussian.

at the wire to $\Delta x \geq 1 \mu \mathrm{m}$. The inter-site separation in the optical lattice is approximately $\lambda=400 \mathrm{~nm}$, but can be increased to $1 \mu \mathrm{m}$ by setting an angle between the counter-propagating lasers in such a way that $\Delta x \leq \lambda$. The variation of the magnetic field at the wire can be adapted to typical Feshbach resonance widths, which are of the order of $1-10 \mathrm{mG}$. Fig. 1 shows an example of the variation of the on-site interaction for a typical experimental setup.

Under these conditions, the system is described by a Bose-Hubbard Hamiltonian with random interatomic interactions:

$\hat{H}=-J \sum_{<i j>}\left(b_{i}^{\dagger} b_{j}+\right.$ h.c. $)+\sum_{i} \frac{U_{i}}{2} n_{i}\left(n_{i}-1\right)-\sum_{i} \mu_{i} n_{i}$,

where $b_{i}\left(b_{i}^{\dagger}\right)$ is the annihilation (creation) operator for bosons in the $i$-th lattice site, $n_{i}=b_{i}^{\dagger} b_{i}, J$ is the hopping constant, $\mu_{i}=\mu$ is the local chemical potential, and $U_{i}$ denotes the local value of the coupling constant for the interatomic interactions. We assume $U_{i}$ uniformly distributed inside the range $U(1-\epsilon) \leq U_{i} \leq U(1+\epsilon)$ [18.

In order to assess the effects of randomness in the on-site interactions and to contrast it to the case of a random chemical potential, we shortly review the latter case, where $\mu-\Delta \leq \mu_{i} \leq \mu+\Delta$, at a fixed $U_{i}=U$ $(\epsilon=0)$ [12]: The phase diagram in the strongly interacting regime can be obtained from considering first the trivial limit, $J=0$ : One finds that if $\mu$ falls within an interval $(n-1) U+\Delta \leq \mu \leq n U-\Delta$, with an integer $n$, the ground-state of the system is an incompressible Mott insulator with $n$ bosons on each lattice site. For values of $\mu$ outside these intervals, i.e. for $n U-\Delta<\mu<n U+\Delta$, the system enters an insulating, but compressible phase commonly referred to as Bose glass, in which the occupa- tion per site varies between $n$ and $n+1$. This physics is maintained up to moderate values of $J$, leading to a phase diagram with three distinguished phases: superfluid (SF) at large $J$, Bose glass (BG), and the Mott insulator (MI) regions. Recent quantum Monte Carlo calculations have shown that the transition from SF to MI always occurs through an intermediate BG phase [19], as suggested in Ref. [12].

A characteristic difference to the case of random $U$ at a fixed value of $\mu_{i}=\mu(\Delta=0)$, is already obtained in the limit $J=0$ : Now compressible phases occur for $n(1-\epsilon)<\mu / U<n(1+\epsilon)$, in which the occupation per site varies between $n$ and $n+1$, whereas outside these regions the system enters into a MI. In contrast to the case of randomness in the chemical potential, the extent of compressible regions between the MI thus increases with the occupation number $n$. The disorder in $U$ thus leads to an increased destabilization of MI states with increasing $n$. Eventually, MI regions with an occupation of $n \geq(1+\epsilon) / 2 \epsilon$ particles per site disappear. Therefore, given a disorder strength $\epsilon$, only a finite number of MI regions remain stable. This selective destruction of MI states cannot be accessed in the case of a random chemical potential, where all the MI lobes vanish once $\Delta \geq U / 2$. More generally, if both $U_{i}$ and $\mu_{i}$ have a bounded random distribution, the $n$-th lobe disappears for $(2 n-1) \epsilon+2 \Delta / U=1$.

In order to analyze the phase diagram for finite hopping $J>0$, we first estimate the extent of the MI regions using the SCE 20]. This method allows for a quantitative calculation of the boundaries between compressible and incompressible phases for sufficiently low $J$, particularly in low dimensions, where the coordination number is small.

From the SCE one obtains the energy gap for adding or removing a particle from the MI, and performs an expansion in the small parameters $J / U, \Delta / U$ and $\epsilon$. For a given value of $J$, these gaps determine the boundaries of the MI lobes, which are obtained in first order as

$$
\begin{aligned}
& \frac{\mu_{u}}{U}=-2(n+1) \frac{J}{U}-\frac{\Delta}{U}+n(1-\epsilon) \\
& \frac{\mu_{l}}{U}=2 n \frac{J}{U}+\frac{\Delta}{U}+(n-1)(1+\epsilon)
\end{aligned}
$$

for the upper $(u)$ and lower $(l)$ boundaries of the MI with integer filling $n$. In particular we find, that the lower boundary of the first MI lobe $(n=1)$ is not affected by the disorder in $U$, in contrast to the random- $\mu$ case [20]. This reflects the observation, that no compressible phase emerges below the $n=1 \mathrm{MI}$ at $J=0$ for the random- $U$ case, in contrast to the case of random $\mu$.

From equating the two boundaries one obtains an (under-) estimate for the largest extent of the MI phase with filling $n$ :

$$
\frac{J_{c}}{U}=\frac{1-2 \Delta / U-(2 n-1) \epsilon}{2(2 n+1)} .
$$


Calculating higher-order terms in the strong-coupling expansion improves the quantitative description of the MI lobes, but does not affect our qualitative conclusions. In particular, all the higher order terms in the random interaction are at least of order $(J / U)^{2} \epsilon$. Considering that the critical hopping $J_{c} / U \ll 1$, even relatively large disorder strengths may be approximated using the first order shift of the phase boundaries. For the figures presented below, we used expansions up to third order and also considered finite size effects [20]. The latter, as shown below, are rather significant, and converge only very slowly to the thermodynamic limit when the number of lattice sites considered increases.

While the SCE allows to estimate the extent of the Mott-insulating phases quantitatively, it does not provide information about the complete phase diagram, in particular concerning the nature of the compressible phases and the presence of SF and BG phases. In order to obtain a more complete phase diagram for bosons with random on-site interactions we have performed QMC simulations using the stochastic series expansion method 21] with directed loop updates [22, 23]. We performed simulations for periodic chains with $L=200$ sites averaged over typically 200 disorder realizations for each data point. In the simulations, the temperature $T$ was chosen low enough to obtain ground state properties of the finite systems [24]. In order to reliably identify the various phases of the system, we measured the global compressibility $\kappa=\partial N / \partial \mu$ from the fluctuations of the particle number $N$, and the winding number fluctuations $\left\langle W^{2}\right\rangle$, from which the superfluid density is obtained as $\rho_{S}=L T /(2 J)\left\langle W^{2}\right\rangle$ 25]. Fig. 2 shows the disorder averaged values of both observables as functions of $J / U$ along a cut of constant $\mu / U=0.51$ for $\epsilon=0.25$, which at $J=0$ belongs to the MI region with $n=1$. At low values of $J$, an extended MI region with $n=1$ is clearly identified by vanishing values of both $\kappa$ and $\rho_{S}$. For large value of $J / U$, both $\kappa$ and $\rho_{S}$ take on finite values, thus identifying the large$J$ region as a SF phase. In the intermediate region, for $0.078 \lesssim J / U \lesssim 0.133$, the system shows a finite compressibility, but no superfluid response. This regime is thus characterized as a disorder-induced BG phase, separating the MI and SF region at this value of $\mu / U=0.51$.

Performing similar scans along different lines in parameter space, we obtain QMC estimates of the various phase boundaries, leading to the phase diagram of Fig. 3 Here, we combined the QMC results for $\epsilon=0.25$ with third-order SCE results for the extent of the MI phases in the thermodynamic limit (TDL). Within the chosen resolution, the finite size corrections of the phase boundaries obtained by QMC show only a weak dependence on the system size. An accurate determination of the MI boundaries from QMC in the disordered case [26] would require significantly larger system sizes due to rare regions of delocalized bosons [20]. In fact, performing a third-order SCE for a finite chain of $L=200$ sites com-

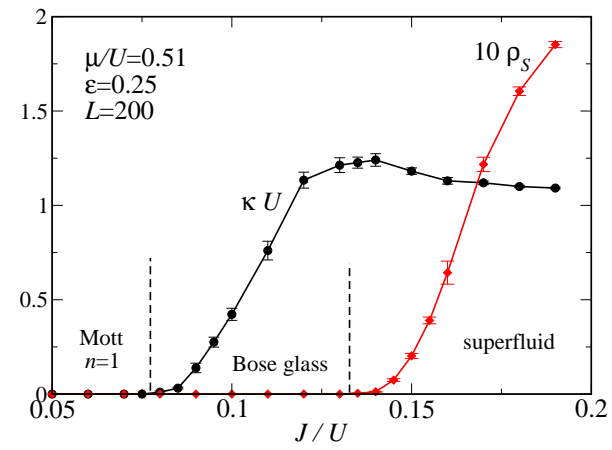

FIG. 2: Zero temperature compressibility $\kappa$ and superfluid density $\rho_{S}$ of bosons on a one-dimensional optical lattice with random interaction strength of $\epsilon=0.25$ as a function of $J / U$ along a cut a $\mu / U=0.51$, obtained from QMC simulations. The error bars quantify the standard deviation of the sampleto-sample fluctuations, whereas the dashed lines indicate the estimated extent of the Bose glass along this cut.

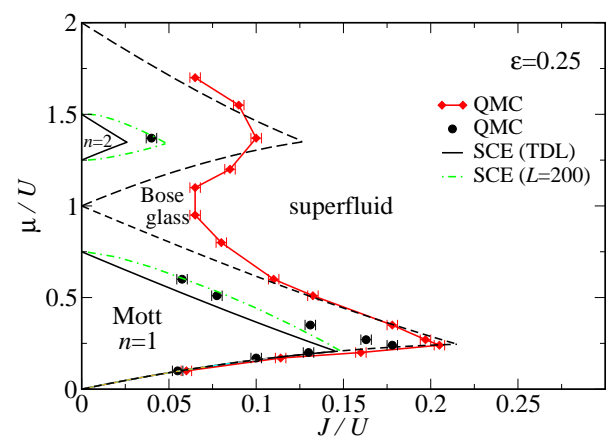

FIG. 3: Zero-temperature phase diagram of bosons on a onedimensional optical lattice with random interaction strength of $\epsilon=0.25$, obtained from QMC simulations of 200 sites and a third-order SCE for the thermodynamic limit (TDL). The extent of the Mott lobes in the pure case $(\epsilon=0)$ from SCE is indicated by the dashed line, whereas the dot-dashed line shows the SCE results for a finite system of $L=200$ sites.

pares well to the estimated extend of the MI regions from QMC (Fig. 13) in the strong-coupling regime, $J / U \lesssim 0.1$, and indicates the rather strong finite size effects in the disordered case.

Our numerical calculations confirm the qualitative picture considered above: The MI lobes clearly shrink, not only in the $\mu$-direction but also in $J$, and eventually disappear for sufficiently large values of $\mu$. In the case considered in Fig. 3 where $\epsilon=0.25$, only the MI lobes with $n=1$ and 2 remain stable. Furthermore, we observe that the lower boundary of the $n=1 \mathrm{MI}$ does not vary significantly with the disorder strength up to the critical hopping $J_{c}$. The reduced relevance of disorder in the interaction strength in the low-density region is also reflected by the complete absence of a BG phase for $\mu<0$. Therefore, a dilute compressible lattice boson gas remains superfluid even in the presence of disordered interactions. This is in clear contrast to the case of random 
$\mu$, where the BG extends down to arbitrary low but finite densities. The absence of a BG for $\mu<0$ indicates a tricritical point for the $\mathrm{SF}, \mathrm{MI}$, and $\mathrm{BG}$ phases along the lower boundary of the $n=1 \mathrm{MI}$. However, our calculations cannot reliably distinguish whether the BG phase disappears exactly at a tricritical point at $\mu=J=0$, or if this occurs for finite values of $\mu, J>0$. This interesting question thus remains open for future studies.

To summarize, in this Letter we analyzed the novel physics occurring when the on-site interaction in a BoseHubbard Hamiltonian acquires a bounded random character. We discussed how this exciting possibility can be realized in ultracold atomic gases in optical lattices at the verge of a Feshbach resonance, when they are brought in the vicinity of weak spatially random magnetic fields generated by magnetic wires. As for the case of random chemical potentials, a phase diagram with superfluid, Bose-glass and Mott-insulator phases is predicted. However, important differences can be found when comparing the random $U$ and random $\mu$ cases: For random interactions, the Mott-insulator phases become progressively narrower (both in the $\mu$ and $J$ direction) for larger occupation numbers, and eventually disappear beyond a given filling. Furthermore, the Bose-glass phase disappears for low chemical potentials and hopping, and hence a tricritical point occurs, although our numerical calculations cannot discern whether this occurs at $\mu=J=0$ or very small but finite values.

Our calculations were performed in absence of an overall (usually harmonic) confining potential, whereas the necessary commensurability for Mott-insulator phases can be achieved in practice only in the presence of inhomogeneous potentials. However, if the confining potential is sufficiently shallow, a local chemical potential may be considered, and local phase occur, as thoroughly demonstrated by means of QMC calculations [27, 28] for the pure case. Of course, the finite size of these different regions could significantly affect the boundaries between the Bose glass and superfluid, once the localization length becomes smaller than the spatial extent of the various regions. Typical experiments on the Mott-insulator phase of atoms in optical lattices 4, 5] rely on the observation of the broadening of interference fringes in timeof-flight pictures (provided by the insulating character of the phase), and the monitoring of the opening of the Mott-gap in the excitation spectrum by means of tilting experiments. In these experiments, although other spatial phases are expected, the Mott-insulator region is the largest one and dominates the experimental detection. Similar experiments could be performed to detect the shrinking of the Mott-insulator phases as a function of the disorder in $U$ predicted in this paper.

Fruitful conversations with A. Kantian, M. Lewenstein, and P. Öhberg are acknowledged. This work was supported by the Alexander von Humboldt Foundation and by NIC at FZ Jülich. H.G. thanks the Studienstiftung des deutschen Volkes for support.

[1] M. H. Anderson, et al.,Science 269, 198 (1995).

[2] B. DeMarco and D. S. Jin, Science 285, 1703 (1999).

[3] C. A. Regal, M. Greiner, and D. S. Jin, Phys. Rev. Lett. 92, 040403 (2004); M. W. Zwierlein, et al., Phys. Rev. Lett. 92, 120403 (2004).

[4] M. Greiner et al., Nature 415, 39 (2002).

[5] T. Stöferle, H. Moritz, G. Schori, M. Köhl and T. Esslinger, Phys. Rev. Lett. 91, 130403 (2004).

[6] B. Paredes et al., Nature 429, 277 (2004).

[7] L. Santos et al., Phys. Rev. Lett. 93, 030601 (2004).

[8] S. Wessel and M. Troyer, cond-mat/0505298

[9] B. Paredes, C. Tejedor, and J. I. Cirac, cond-mat/0306497

[10] B. Damski et al., Phys. Rev. Lett., 91, 080403 (2003); R. Roth and K. Burnett, J. Opt. B Quant. Semiclass. Opt. 5, S50 (2003).

[11] P. W. Anderson, Phys. Rev. 109, 1492 (1958).

[12] M. P. A. Fisher et al., Phys. Rev. B 40, 546 (1989).

[13] J. E. Lye, et al., cond-mat/0412167

[14] A. Sanpera, et al., Phys. Rev. Lett. 93, 040401 (2004).

[15] E. Tiesinga et al., Phys. Rev. A 47, 4114 (1993); S. Inouye et al., Nature (London) 392, 151 (1998).

[16] In free space, a renormalization group analysis shows that the critical properties at the transition between superfluid and disordered state are unaltered as compared to the case of random $\mu$. A. Kantian, Dipl. Thesis, Univ. Hannover (2004), and private communication.

[17] S. Wildermuth et al., Nature 435, 440 (2005).

[18] As observed in Fig. 11 in practice the distribution of $U_{i}$ is neither ideally symmetric nor uniform, but this does not affect our main conclusions and could be easily included in both the SCE and QMC calculations. Also some residual noise correlations could appear between nearest neighbors, but they are small (20\% in Fig. 1) and can be reduced by increasing the lattice spacing.

[19] N. Prokof'ev and B. Svistunov, Phys. Rev. Lett. 80, 4355 (1998); S. Rapsch, U. Schollwöck, and W. Zwerger, Europhys. Lett. 46, 559 (1999); N. Prokof'ev and B. Svistunov, Phys. Rev. Lett. 92, 015703 (2004).

[20] J. K. Freericks and H. Monien, Phys. Rev. B 53, 2691 (1996).

[21] A.W. Sandvik, Phys. Rev. B 59, R14157 (1999).

[22] O. F. Syljuåsen and A. W. Sandvik, Phys. Rev. E 66, 046701 (2002).

[23] F. Alet, S. Wessel, and M. Troyer, Phys. Rev. E 71, 036706 (2005).

[24] We found a value of $T=0.015 J$ to be sufficient for the considered system sizes.

[25] E.L. Pollock and D.M. Ceperley, Phys. Rev. B 36, 8343 (1987).

[26] R. T. Scalettar, G. G. Batrouni, and G. T. Zimanyi, Phys. Rev. Lett. 66, 3144 (1991).

[27] M. Rigol, A. Muramatsu, G. G. Batrouni, and R. T. Scalettar Phys. Rev. Lett. 91, 130403 (2003).

[28] S. Wessel, F. Alet, M. Troyer, and G. G. Batrouni, Phys. Rev. A 70, 053615 (2004). 